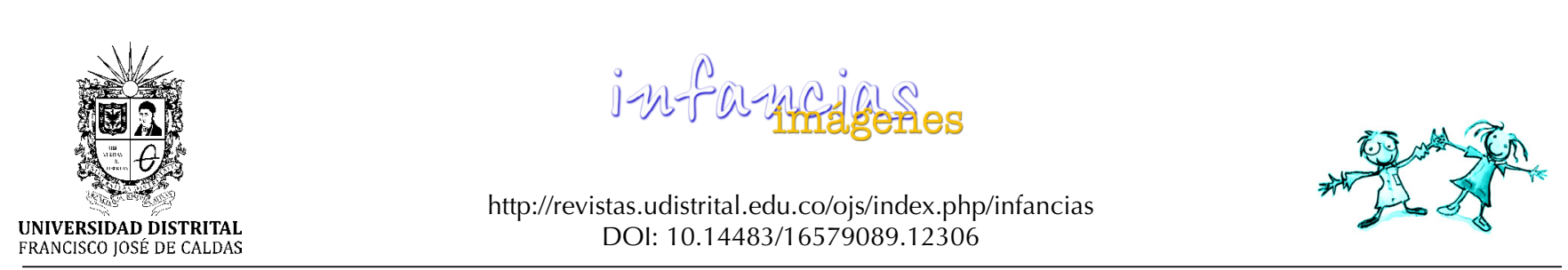

TEXTOS Y CONTEXTOS

\title{
Creencias sobre educación inicial en colegios oficiales de Bogotá* $^{2}$
}

\author{
Beliefs about Early Childhood Education in Official Schools of Bogotá \\ Graciela Fandiño Sandra Marcela Durán ${ }^{2}$ Jenny Maritza Pulido ${ }^{3}$ Erika Liliana Cruz $^{4}$
}

Para citar este artículo: Fandiño, G., Durán, S. M., Pulido, Recibido: 27-enero-2017 / Aprobado: 09-noviembre-2017

J. M. y Cruz, E. L. (2018). Creencias sobre educación inicial en colegios oficiales de Bogotá. Infancias Imágenes, 17(1), 100-108.

\section{Resumen}

Se presentan avances de la investigación "Creencias sobre educación inicial en estudiantes de la Maestría de Estudios en Infancias y egresadas de la Licenciatura en Educación Infantil de la Universidad Pedagógica Nacional". El objetivo de la investigación fue identificar y analizar las creencias que tienen estudiantes y egresadas de estos programas académicos sobre educación inicial, con la intención de reconocer fortalezas y obstáculos en la perspectiva del potenciamiento de desarrollo, la cual orienta el Lineamiento Pedagógico y Curricular para la Educación Inicial en el Distrito (2010), a través de la realización de grupos focales de discusión inscritos en el enfoque cualitativo del proyecto. Los problemas relevantes están referidos al reconocimiento del carácter de la educación inicial, el lugar de las maestras, las características de los niños y las condiciones físicas, ambientales e institucionales que los centros educativos tienen para la realización de este trabajo.

Palabras clave: primera infancia; pensamiento; maestra; educación.

\begin{abstract}
The advances of research "Creencias sobre educación inicial en estudiantes de la Maestría de Estudios en Infancias y egresadas de la Licenciatura en Educación Infantil de la Universidad Pedagógica Nacional" are presented. The research aimed to identify and analyze the beliefs of students and graduates of these academic programs about early childhood education, with the purpose of recognizing strengths and obstacles in the perspective of development empowerment, Which orients the Lineamiento Pedagógico y Curricular para la Educación Inicial en el Distrito (2010), through focus groups of discussion registered in qualitative approach of the project. The relevant problems are related to recognizing early education character, the position of teachers, the characteristics of children and the physical, environmental and institutional conditions of educational centers for realizing this work.
\end{abstract}

Keywords: early childhood; thought; magister; education.

\footnotetext{
* Este artículo presenta avances de la investigación "Creencias sobre Educación Inicial en Estudiantes de la Maestría en Estudios e Infancias y egresadas de la Licenciatura en Educación Infantil de la Universidad Pedagógica Nacional“ (DSI 436 - 16), avalada y financiada por el Centro de Investigación de la Universidad Pedagógica Nacional (UPN), área: Ciencias sociales; subárea: Ciencias de la Educación. Comenzó a desarrollarse desde el mes de marzo de 2016 y concluyó en marzo de 2017.

1 Doctora en Filosofía y Ciencias de la Educación, Uned (España). Profesora asociada, UPN. Coordina el grupo de investigación Educación infantil, pedagogía y contextos. Correo electrónico: gmfandino@pedagogica.edu.co

2 Doctora en Educación Social, Universidad de Granada (España), magister en Educación, especialista en Gerencia Social de la Educación y licenciada en Educación Infantil de la UPN. Profesora de la UPN. Correo electrónico: smduran@pedagogica.edu.co

3 Magister en Educación (UPN) y licenciada en Educación Preescolar. Profesora de la UPN. Correo electrónico: jepulido@pedagogica.edu.co

4 Magister en Educación y Desarrollo Humano, especialista en Investigación Social y licenciada en Educación Preescolar. Profesora de la UPN. Correo electrónico: elcruzv@pedagogica.edu.co
} 


\section{Introducción}

Los resultados preliminares presentados acá tienen su origen en la investigación "Creencias sobre educación inicial en cuatro instituciones educativas distritales", en la cual se indagó por el sentido de la educación inicial con maestras de esta, directivas y docentes de otros grados de básica primaria a partir de tres interrogantes relacionadas con: las finalidades, propósitos y objetivos de la educación inicial; los contenidos y orientaciones curriculares del trabajo en este ciclo particular; y las principales formas de trabajo pedagógico que utilizan los maestros y maestras con las niñas y los niños.

Como resultados preliminares se identificaron entre los docentes dos posturas discursivas disimiles, pero que confluyen en sus prácticas. Por una parte, los procesos de promoción intencional del desarrollo respetando las características y las particularidades de niños y niñas; $y$, por otra, la necesidad que les apremia de prepararlos ante las demandas que le serán exigidas en grados posteriores, principalmente los aprendizajes de contenidos formales sobre operaciones de aritmética básica y la enseñanza alfabética de la escritura y la lectura.

Es este marco, el trabajo en mención ofreció un panorama general en relación con el sentido de la educación inicial que circula en algunas instituciones educativas que cuentan con el ciclo inicial. No obstante, se calcula que son aproximadamente 270.000 niños y niñas entre 3 y 5 años los que actualmente se encuentran en colegios oficiales en la ciudad de Bogotá, lo cual, para el equipo de investigación, merece un análisis más profundo respecto a los sentidos, contenidos y formas de trabajo pedagógico que se dinamizan en estas instituciones. A partir de esto, resultó altamente relevante ahondar en la caracterización de las creencias que frente al sentido de la educación inicial tienen para este caso estudiantes de la Maestría en Estudios en Infancias de la Universidad Pedagógica Nacional (UPN), todas ellas maestras en ejercicio de la educación inicial, algunas egresadas de las licenciaturas en Educación Preescolar e Infantil de la misma institución. Esto con el fin de aportar elementos de análisis claves en relación con el campo formativo y profesional de los maestros de educación infantil.

\section{Problema de investigación}

La integración generalizada de niños y niñas menores de 5 años a las instituciones educativas del distrito se remonta al año 2012, cuando la Administración Distrital se propuso como meta atender de manera integral durante ese cuatrienio a 270.000 niños y las niñas de primera infancia. En este marco, la Secretaria de Integración Social amplió la oferta y cobertura a 210.000 niños y niñas de 0 a 3 años, en dos modalidades de atención integral: atención integral en ámbito familiar y atención integral en centros Acunar. Por su parte, la Secretaría de Educación Distrital (SED), a través del programa 901, creó cerca de 60.000 nuevos cupos para niños y niñas de 3 a 5 años, para lo cual fue necesario adecuar e instalar aulas en colegios oficiales, arrendar inmuebles y planificar la construcción de 190 jardines infantiles. Ello en el marco del componente infraestructura del proyecto, orientado a la "construcción y adecuación de equipamientos adecuados, seguros, acogedores, accesibles, así como el enriquecimiento de otros escenarios como ludotecas" (SED, 2013, p. 24).

Este desafío de cobertura trajo consigo importantes tensiones a las instituciones educativas que asumieron dicha apertura de matrícula y que vale la pena develarlas para comprender cómo este proyecto ha impactado a las comunidades educativas. Dichas tensiones no solamente están relacionadas con temas de infraestructura, pues también les aqueja los procesos pedagógicos que se debaten entre la perspectiva de potenciamiento del desarrollo, en el sentido que sustenta el Lineamiento Pedagógico y Curricular para la Educación Inicial en el Distrito (2010), y por otro lado, el fuerte convencimiento que persiste respecto a la necesidad de preparar y dotar de contenidos a los niños y niñas de la primera infancia para un mejor proceso de transición, adaptación y rendimiento en los primeros grados de básica. Una tensión que viene dada por la misma legislación vigente: Ley General de Educación, Ley 115 de 1994 y Ley 1098 de 2006, Código de la Infancia y la Adolescencia. Este último ha recogido y a la vez posibilitado la política de primera infancia en el país que se desarrolló durante la anterior década y que recientemente se ha plasmado en la Ley 1804 del 2 de agosto de 2016. 
El contexto descrito generó el interés por visibilizar la necesidad de un adecuado acompañamiento pedagógico a los colegios oficiales que han comenzado el proceso de integración de niños y niñas de 3 y 4 años, además de las condiciones necesarias en términos de infraestructura y dotación para la atención integral a la primera infancia.

Para tales efectos, se desarrolló una investigación con las estudiantes de la maestría ya citada. Esto con el propósito, por una parte, de ampliar el objeto poblacional a 48 maestras aproximadamente que trabajan con el ciclo inicial en colegios oficiales, y por otra, dinamizar procesos investigativos que apunten a la articulación de los programas de pregrado y posgrado de la UPN. Lo anterior en la perspectiva de analizar las directrices administrativas de la ciudad de Bogotá en escenarios considerados privilegiados para la problematización de los lugares desde los cuales ha sido y es pensada la educación inicial.

Así entonces, las preguntas que guiaron el estudio según el problema enunciado fueron:

¿Cuáles son las finalidades, propósitos y objetivos que las estudiantes de la Maestría en Estudios en Infancias y egresadas de las licenciaturas en Educación Infantil y Educación Preescolar de la UPN ubican para la educación inicial?

¿Cuáles son los contenidos del trabajo que estudiantes de la Maestría en Estudios en Infancias y egresadas de las licenciaturas en Educación Infantil y Educación Preescolar de la UPN desarrollan en este ciclo particular?

¿Cuáles son las principales formas de trabajo pedagógico que las estudiantes de la Maestría en Estudios en Infancias y egresadas de las licenciaturas en Educación Infantil y Educación Preescolar de la UPN utilizan con las niñas y los niños de cada institución?

¿Cuáles son las principales fortalezas y obstáculos que perciben las estudiantes de la Maestría en Estudios en Infancias y egresadas de las licenciaturas en Educación Infantil y Educación Preescolar de la UPN para el trabajo con las niñas y los niños de cada institución?

A partir del panorama expuesto se propuso como objetivo general del proyecto: identificar y analizar las creencias que sobre educación inicial tienen estudiantes de la Maestría en Estudios en Infancias y egresadas de las licenciaturas en Educación Infantil y Educación Preescolar de la UPN. Para tal efecto se recurrió a la realización de grupos focales de discusión, con la intención de reconocer fortalezas y obstáculos en el enfoque de potenciamiento de desarrollo.

\section{Perspectiva teórica}

Si se concibe que la educación inicial posee un sentido en sí misma y, por ende, unas apuestas pedagógicas que la particularizan del sistema educativo, tanto por los importantes procesos que en esta suceden para el desarrollo humano (en los que se constituyen las bases de la autonomía, la identidad y las capacidades intelectuales y sociales de los niños y niñas), como el desarrollo profesional de los docentes en estos primeros grados (quienes hacen posible que este tránsito por tan importante ciclo vital tenga un protagonismo en la sociedad), podría comprenderse que este tipo de educación es un campo de conocimiento reciente. Lo anterior requiere de profundos desarrollos analíticos que permitan, entre otras cosas, indagar por el sentido con el que se orientan las prácticas de los maestros de este nivel y las formas cómo constituyen en su trayectoria profesional un importante acumulado de saberes a partir de su experiencia, este les permite desenvolverse ante los múltiples retos y desafíos que esta educación plantea.

Desde este punto de vista, la investigación se estructura a partir del concepto de creencia, desarrollado dentro del paradigma de pensamiento del profesor, de tradición anglosajona y que se ha venido exponiendo a través de anteriores trabajos (Fandiño, Castaño y Rojas, 2006; Durán, 2012; Martín, Pulido y Durán, 2012). Como investigadoras formadoras de maestros, se considera fundamental indagar por las creencias de los profesores, pues a como lo indica Gary Fenstermacher (1989), estas son relevantes para comprender su práctica. "La investigación influye sobre la práctica cuando altera la verdad o falsedad de las creencias que el profesor tiene, cuando cambia la naturaleza de esas creencias y cuando añade creencias nuevas" (p. 165).

A su vez, con la necesidad de ampliar el concepto de creencia con otras categorías provenientes 
de campos de estudio de las Ciencias Sociales que han indagado sobre la construcción del conocimiento docente, se extiende la mirada al concepto de saber y más específicamente con el concepto de saber de la experiencia.

Desde perspectivas más amplias de las Ciencias Sociales el saber se concibe, según Moreno, Rodríguez de Moreno, Torres, Mendoza y Vélez, "como una práctica discursiva, es decir, un conjunto de enunciados y de procedimientos socialmente constituidos" (2006, p. 23) que se relacionan entre sí, como consecuencia de lo que se produce en un acumulado enriquecido de experiencias y que se manifiestan en actuaciones consientes.

\section{El concepto de saber}

Para comprender esta perspectiva resulta preponderante retomar a Moreno et al. (2006), quienes siguiendo a Jean-François Lyotard plantean que el saber es la forma más amplia de intelección humana, los "seres humanos accionamos por el saber y no por el conocimiento", ya que el saber está fuertemente arraigado a una dimensión cognoscitiva, ética, estética y axiológica, según las finalidades que lo dinamicen. Desde esta perspectiva, la concepción de saber toma distancia de la noción de una forma de entendimiento pasivo, no reflexivo, sin veracidad o no sistemático. Por el contrario, se instaura al saber cómo antecesor y condición necesaria para el desarrollo del conocimiento. En este sentido, en escenario en el que es posible la duda, la pregunta, la desnaturalización de las certidumbres y las evidencias

Por su parte, Jacky Beillerot (1996, citada por Mancoksky, 2009) establece una clara distinción entre saber, en singular; saber, en plural, y saber-hacer. La primera es entendida como el saber constituido y enriquecido por la experiencia y como resultado de un proceso de aprendizaje, el cual no es estático, sino que puede ser actualizado y en menos oportunidades trasformado al ponerse en evidencia y en situación real de contexto en situaciones y en prácticas que requieran este saber.

Con el saber en plural, Beillerot se refiere a un conjunto de enunciados y procedimientos socialmente constituidos y reconocidos (1998, p. 17). Por medio de este tipo de saberes es posible que el sujeto establezca relaciones y afecte su contexto. Así, el saber es el que un determinado grupo social es capaz de constituir, formalizar, sistematizar, plantear, empoderarse en él y transmitirlo, en otras palabras, legitimarlo; puede ser con un carácter ético, estético, institucional, profesional, social o político. En la construcción de un saber el sujeto hace uso de sus intuiciones, percepciones propias de su ciclo vital y género, sus experiencias previas, su legado cultural, en fin, de lo que le constituye como sujeto histórico. No obstante, estos saberes en interacción o en relaciones dominantes con otros pueden ser transformados o revestidos de nuevos sentidos.

Por otro lado, como lo indican Moreno et al. (2006), los sujetos construyen un saber respecto a las formas de actuar con otros y de resolver variadas situaciones en contexto, es decir, la manera como en la vida cotidiana se sortean y resuelven situaciones, problemas, dilemas y tensiones. De esta manera se dispone en escena lo aprendido, es decir, hace visible un saber hacer que permite acumular en la experiencia un repertorio de posibilidades actuativas, que a su vez se pueden convertir en plataforma de transformación cuando son expuestas a tensión o desequilibrio.

En este sentido y siguiendo a Moreno et al. (2006), el saber se expresa en la acción, algunas veces reflexionada, otras de manera más intuitiva, en cuya construcción interviene lo particular de cada individuo, pero también lo sociocultural, esto es, la relación intersubjetiva que se construyen los diversos ámbitos. Específicamente, y en lo que atañe a esta investigación, para comprender los saberes que le son propios al educador infantil resulta importante resaltar precisamente el lugar de la experiencia como saber que posibilita la seguridad de desenvolverse en su campo profesional, el dominio de ciertas rutinas, las certezas de la implementación de metodologías y las apropiaciones discursivas con las que puede enunciarse y ampliar sus horizontes de sentido.

En el ámbito educativo, la tensión permanente o división entre el saber del docente y el conocimiento pedagógico ha sido un campo de importantes investigaciones que han permitido establecer marcos de comprensión sobre el tipo de saber que 
permea las prácticas de enseñanza de los docentes. Siguiendo a Blanco (2015), las maestras y los maestros desarrollan saberes propios en el contexto de sus prácticas. Saberes que a veces están en relación con los generados por la investigación sobre la educación, algunas veces en abierta contraposición a ellos y otras sin tenerlos en consideración, ya sea porque han sido impuestos y han generado socialmente un rechazo.

También, en el plano estricto de los saberes docentes, es importante referirse al sociólogo Maurice Tardif (2009), quien toma distancia de la perspectiva anglosajona, por considerarla "mentalista" e individualista. Para el autor, el saber de los maestros está relacionado con "las realidades sociales, organizativas y humanas en la que se encuentran inmersos los educadores" (p. 10). En ese sentido, el saber de los maestros es un saber social, aunque sin desconocer que es el saber de ellos.

Para Tardif, este saber es social porque es compartido con otros profesores con los que tienen una formación común y trabajan en una misma organización. Su posesión y utilización descansa sobre un sistema que garantiza su legitimidad; lo que un profesor debe enseñar no es un problema epistemológico, sino una cuestión social. Sus propios objetos son prácticas sociales, relaciones complejas entre maestros y alumnos. Evolucionan con el tiempo y los cambios sociales. Es un proceso de construcción a lo largo de un recorrido profesional. En ese sentido, y distanciándose también de un sicologismo, afirma:

[...] el saber del profesorado no es el "foro" íntimo poblado de representaciones mentales, sino un saber siempre ligado a una situación de trabajo con otros (alumnos, padres, etc.), un saber anclado en una tarea compleja (enseñar), situado en un espacio de trabajo (aula, escuela), enraizado en una institución y en una sociedad. (Tardif, 2009, p. 13)

En esta línea de los saberes docentes como construcción social, según una perspectiva latinoamericana, es importante retomar a Ruth Mercado (2002), quien parte, entre otros, de los desarrollos teóricos de la sociología de la vida cotidiana de Agnes Heller y de la visión antropológica de Clifford
Geertz, y utiliza el concepto de saberes docentes, los cuales caracteriza como pluriculturales, históricos y socialmente construidos. Son saberes de los que el maestro se apropia en tanto le son necesarios para su trabajo en el aula. Estos se construyen en interacción con los alumnos, los materiales curriculares, los colegas, los padres y demás elementos que le llegan a la escuela o que se producen dentro de ella relacionados con la enseñanza.

Dicha apropiación se fundamenta de manera importante en la reflexividad que la enseñanza misma impone a los maestros [...] En el proceso de apropiarse de este particular conocimiento los maestros generan nuevos saberes a la vez que integran o rechazan propuestas pedagógicas provenientes de distintas épocas y ámbitos sociales. En esta línea en la construcción de este saber. (Mercado, 2002, p. 14)

\section{Saber de la experiencia}

Pero aunado a estos conceptos de saber que enriquecen esta investigación resulta necesario un proceso de profundización teórica sobre la experiencia docente como una categoría conceptual en relación con el conocimiento práctico o tácito que un maestro logra consolidar para desenvolverse en la vida cotidiana de su hacer profesional. Lo anterior, asumiendo que el emerge consustancial a los individuos, a su historia, a lo vivido, a sus relaciones, a sus afectos y sus vínculos. En tal sentido, el COnocimiento de un docente también es el resultado interiorizado de la experiencia individual.

De la misma forma, Blanco (2015) destaca que el trabajo investigativo que durante varias décadas se ha producido en Italia a partir de un movimiento de mujeres y que luego se ha extendido, principalmente en España, ha buscado de manera protagónica conocer los propios saberes de las maestras —en femenino-. Esto para conocer cuáles son los sentidos que orientan la enseñanza y atribuirles un lugar legítimo que trasciende el conocimiento pedagógico formal.

Ese saber que se genera y cobra protagonismo en la vida cotidiana de la escuela ha sido nombrado también por Anna Piussi como "saber que se sabe" (2013, p. 32), por medio del cual se designa "la consciencia de un saber, nacido de la práctica 
política de referencia a otras mujeres y que se produce recurrentemente al interrogarse por el sentido de sí, por la propia acción y por el sentido del mundo" (p. 33).

En esta vía, nos enriquecemos también con José Contreras y Nuria Pérez (2010), quienes retomando a Hannah Arendt afirman que no es posible pensar sin experiencia personal.

Es la experiencia la que pone en marcha el proceso de pensamiento. Pensamos porque algo nos ocurre; pensamos como producto de las cosas que nos pasan, a partir de lo que vivimos, como consecuencia del mundo que nos rodea, que experimentados como propio, afectados por lo que nos pasa. (p. 21)

Es así como reconocen que el saber propio de la experiencia de las maestras se despliega en conexión con las necesidades, los contextos, las historias singulares; por ello, tiene un potencial transformador de las realidades, de la relación de los sujetos consigo mismos, con los otros y con la vida. Es esta condición justamente la que acerca el saber pedagógico al saber de la experiencia, al estar ambos saberes en contacto con las vivencias de los sujetos concretos.

Los autores de la corriente de pensamiento en mención proponen que se retome el saber producido sobre y en la escuela, como un saber de la experiencia que aporta a la investigación educativa (Blanco, 2015; Orozco y Gabbarini, 2014). Al ser un saber dinámico y enriquecido por múltiples variables, que reconoce sus vicisitudes, acoge aspectos expulsados del campo de la ciencia, el saber de la escuela puede aportar a nuevos avances y comprensiones sobre la complejidad del quehacer pedagógico. El saber de la experiencia, así como el saber pedagógico, es un saber en primera persona que actúa desde quien se es y no en representación de quien no se es, y por ello acoge los acontecimientos que nos suceden dentro y fuera del aula.

En este sentido, se puede decir que una investigación del saber de la experiencia de las maestras más que intentar explicar desde afuera lo que ellas piensan o cómo piensan, o porque hacen lo que hacen, intenta dar cuenta de un saber que se construye en relación intersubjetiva y en un contexto situado. Por tanto, es el diálogo el que permite ahondar y develar la trayectoria de aquello que para esa maestra ha significado su práctica, los acontecimientos que han marcado su identidad, su quehacer. En últimas, su decir y su pensar acerca de las relaciones que ella establece con aquello que es su práctica docente, sus efectos y los saberes que de ella logra decantar como experiencia viva y acopiada con un valor único.

\section{Metodología}

La metodología de la investigación está orientada por un enfoque cualitativo, ello en la perspectiva de "comprender la realidad subjetiva que subyace a las acciones de los miembros de la sociedad" (Torres, 1995, p. 3). Desde esta óptica, se pueden estudiar relaciones sociales, analizar prácticas culturales, indagar diferentes puntos de acuerdo con los distintos actores sociales que intervienen en un proceso de investigación y, además, abordar temáticas en las que la dimensión subjetiva y simbólica son decisivas.

Otro aspecto importante en la investigación cualitativa es el desarrollo de una relación estrecha, cooperativa y participativa entre los investigadores y los actores. Esta estructura relacional (Zabalza, 1988) es la que permite comprender el punto de vista del informante, a partir de la validación de las interpretaciones, utilizando procesos de retroalimentación con los mismos actores (Woods, 1987).

Desde este punto de vista, el proyecto de investigación se realiza a partir de grupos focales de discusión, como técnica que se centra en la pluralidad y variedad de las actitudes, experiencias y creencias de los participantes (Martínez, 2004).

Martínez (2004) plantea que, si bien las dos técnicas principales usadas para recoger información en la metodología cualitativa son la observación participativa y las entrevistas en profundidad, los grupos focales poseen elementos de ambas técnicas que, aunque mantienen su unicidad y distinción como técnicas de investigación, son modos de escuchar a las personas y aprender de ellas. Se afirma que los participantes en los mismos encuentran la experiencia más gratificante y estimulante que las entrevistas individuales; además, al confrontar diferentes puntos de vista están expuestos a repensar los propios. 
Población y procedimiento de la investigación Como se mencionó, la población objeto de esta investigación se conforma por estudiantes de maestría y egresadas de la Licenciatura en Educación Preescolar y Educación Infantil de la UPN. Todas ellas son estudiantes en la UPN y trabajan en jardines infantiles, en ciclo inicial o primer ciclo. Esto hizo preciso adaptar los grupos focales, ya que no estaban compuestos solamente por maestras de niños de 3 y 4 años, sino que participaron todas las maestras que trabajan en la educación inicial en general. En total, se realizaron seis grupos focales con un total 13 maestras, uno de ellos compuesto por maestras acompañantes del proyecto 901 y otro por un grupo de reconocido de maestras investigadoras que desde hace varios años realizan investigación de aula ligadas a la red de lenguaje Ilamado Grupo Semillando.

Es preciso advertir que, en el marco de las investigaciones del pensamiento del profesor, para realizar este tipo de estudios se debe contar con la voluntad y disposición de los actores de las mismas, lo que hace que su participación fuera totalmente facultativa.

Los grupos focales tuvieron en promedio entre tres y siete participantes y con una duración aproximada de una hora. En razón a que gran parte de las experiencias de las maestras de educación inicial son compartidas, los grupos fueron homogéneos y se organizaron aleatoriamente, buscando en el desarrollo de cada uno la presencia de maestras de ciclo inicial y primer ciclo.

Al buscar una profundización con el concepto de saber los grupos focales se hicieron con preguntas más abiertas que buscaron comprender desde la óptica de las participantes cómo estaban viviendo el programa. Los grupos focales se dinamizaron con las siguientes preguntas:
- ¿Cómo analizan la situación del ingreso a los niños de 3, 4 y 5 años a la institución educativa?, ¿cómo han vivido este proceso?, ¿qué piensan en relación con esta situación desde su experiencia y sus saberes como maestras?

- ¿ ¿Qué diferencias hay entre las instituciones privadas y públicas?

- ¿Cuál es el papel de las instituciones en la determinación de contenidos de la educación inicial?

- ¿Cuál es el papel de los padres de familia?

- ¿Cuál es el sentido de la educación inicial?

- ¿Cómo son los niños y niñas de educación inicial?

- ¿QQué aspectos se trabajan en educación inicial?

- ¿Cuáles son los principales obstáculos que observan las maestras para el trabajo en educación inicial?

\section{Puntos de llegada}

Para el análisis y la categorización de los grupos de discusión, el grupo parte del análisis de contenido, retomando los planteamientos de Rafael Porlán, Rosa Martín del Pozo y Ana Rivero (1998), Miguel Martínez (2004), Bonilla y Rodríguez Sehk (1997). Para tal efecto, en primer lugar, se transcribieron los grupos focales y se consolidaron protocolos de cada uno. Luego, con cada protocolo se procedió a clasificar los textos por los enunciados de cada maestra y se realizó una primera categorización inductiva por percepciones recurrentes. Como tercera instancia se consolidó un esquema analítico en el cual se construyeron unidades proposicionales o categorías inductivas que les fueran comunes y no comunes a todos los grupos focales.

Tabla 1. Identificación general de la maestra (institución/nombre completo/ edad).

\begin{tabular}{|l|l|}
\hline 1 & Formación académica. \\
\hline 2 & Descripción de trayectoria y experiencia profesional. \\
\hline 3 & Discursos en torno a la conveniencia de la educación inicial. \\
\hline 4 & Posturas en torno a la diferencia de la educación pública y la educación privada. \\
\hline 5 & Posicionamientos con relación con la política educativa para la infancia y sus implicaciones en las instituciones. \\
\hline 6 & Enunciados en torno a las principales dificultades: infraestructura física, espacios y ambientes adecuados y seguros. \\
\hline 7 & Enunciados en torno a la organización curricular y planeación docente. \\
\hline 8 & Defensa y posicionamiento de la profesión docente. \\
\hline
\end{tabular}


Con el fin de elaborar el análisis de la información desde los conceptos de saber y saber de la experiencia, se optó por una categorización que permitiese análisis más globales. Las cuatro grandes categorías inductivas son saberes relacionados con:

1. Nivel social. La estructura económica, social y política en que la institución educativa se inserte.

2. Nivel institucional. Aquí se han de considerar influencias como la administración escolar, el currículum, las y los colegas y los padres de familia.

3. Nivel de clase o nivel didáctico. Los alumnos, así como los elementos estructurales que caracterizan a la enseñanza.

4. Nivel personal. Las maestras como sujetos de experiencia pedagógica personal.

Estas grandes categorías por niveles se asemejan a los trabajados en las investigaciones de maestros principiantes (Fandiño y Castaño, 2014) en los que se indaga por los problemas de enseñanza de los maestros en sus primeros años de trabajo. Una categorización de estas características permite al equipo de investigación partir de unas categorías generales comunes, lo que posibilita el surgimiento de subcategorías propias de cada grupo focal sin buscar una homogenización de las mismas.

Como punto de llegada preliminar, es de destacar que el poder trabajar con maestras experimentadas que hace varios años se desempeñan profesionalmente en educación inicial, quienes además han desarrollado investigación en el aula, son reconocidas por ello y se encuentran realizando estudios de maestría, permite tener una mirada cualificada de los retos de este nivel educativo.

Entre estos, sobresale el reconocimiento de las características de los niños de estas edades y los desafíos que estarían en capacidad de conquistar de acuerdo a sus ciclos vitales de desarrollo. Aquí la investigación permite advertir la imperante necesidad de establecer debates que posibiliten resignificar la dimensión de cuidado, como la posibilidad de pensar, de representarse al otro y como oportunidad para superar la asociación con lo elemental, lo fácil, y en definitiva como una tarea que, o bien escapa al ámbito profesional de los maestros de niños y niñas o en su defecto, lo define. Ello en relación con la apropiación del sentido diferenciador de la educación inicial ante los demás ciclos de formación.

A propósito de ello, con el Lineamiento Pedagógico y Curricular para la Educación Inicial en el Distrito (2010) se emprendió la transición de un modelo educativo preescolar centrado en la preparación para la primaria (primarización) a uno de educación inicial centrado en el potenciamiento del desarrollo. Aunque este viraje se articula a otras transformaciones de fondo en la comprensión que el país tiene acerca de quiénes son los niños en primera infancia, las maestras insisten en que ese debate tan serio no se ha dado.

En este contexto, aunque la mayoría de maestras expresan una apropiación evidente frente a dicho lineamiento y dan cuenta de las transformaciones que este ha traído en la definición de orientaciones pedagógicas, resultan también factores determinantes para su implementación las condiciones de contratación de las maestras, muchas de ellas en condición de provisionalidad, lo que además de interrumpir frecuentemente los procesos pedagógicos de niños incide en su condición profesional y laboral.

De igual manera, se encuentran aspectos referidos a infraestructura, acceso a materiales, el número de niños por grupo, las dinámicas propias de la institución educativa y el establecimiento de relaciones con las familias; asuntos que muchas veces interfieren en el acompañamiento y seguimiento a los niños.

Es lo expuesto un panorama que merece mayores elaboraciones y discusiones referidas a la educación inicial, sus actores, saberes, procesos y escenarios en la perspectiva de contribuir a la producción de conocimiento sobre la misma.

\section{Referencias}

Alcaldía Mayor de Bogotá (2010). Lineamiento pedagógico y curricular para la educación inicial en el Distrito. Bogotá: DVO Universal. Recuperado de http://www.educacionbogota.edu.co/ archivos/Educacion ini

Beillerot, J. (1998). Saber y relación con el saber. Buenos Aires: Paidós. 
Blanco, N. (2015). Reconocer la autoridad femenina en la educación: Ios saberes de las maestras. Qurriculum, 28, 11-31. Recuperado de http://qurriculum.webs.ull.es/wp-content/ uploads/2015/04/01-Nieves-Blanco.pdf

Bonilla, E. y Rodríguez, P. (1997). Más allá del dilema de los métodos. Bogotá: Norma.

Contreras, J. y Pérez, N. (2010). Investigar la experiencia educativa. Madrid: Morata.

Durán, S. (2012). Los rostros y las huellas del juego (tesis doctoral). Universidad de Granada, España.

Fandiño, G., Castaño, I. E. y Rojas, S. L. (2006). Creencias sobre la articulación preescolar primaria. Informe de investigación. Bogotá: Centro de Investigaciones de la Universidad Pedagógica Nacional (CIUP).

Fandiño, G. y Castaño, E. (2014). Haciéndose maestras: problemas de enseñanza en su primer y tercer año de trabajo de las maestras de Educación Infantil. Bogotá: CIUP, Universidad Pedagógica Nacional, Editorial Magisterio.

Fenstermacher, G. (1989). Tres aspectos de la filosofía de la investigación sobre la enseñanza. En M. C. Wittrock, La investigación de la enseñanza, I. Enfoques, teorías y métodos (pp. 150175). Barcelona: Paidós.

Mancoksky, V. (2009). ¿Qué se espera de una tesis de doctorado? Breve introducción sobre algunas cuestiones y expectativas en torno a la formación doctoral. Revista Argentina de Educación Superior, 1(1), 201-216.

Martín, C., Pulido, J. y Durán, S. (2012). Creencias de los docentes de tres jardínes infantiles de la Secretaría de Integración Social respecto a las rutinas en la educación inicial. Informe de Investigación (DSI-318-12). Bogotá: CIUP, Universidad Pedagógica Nacional.

Martínez, M. (2004). Los grupos focales de discusión como método de investigación. Heterotopia. Recuperado de http://es.slideshare. net/9472326/los-grupos-focales-de-discusin
Mercado, R. (2002). Los saberes docentes como construcción social. México: Fondo de Cultura Económica.

Moreno, N. P., Rodríguez, A., Torres , J. C., Mendoza, N. C. y Vélez, L. (2006). Tras las huellas del saber pedagógico. Bogotá: Universidad Pedagógica Nacional.

Orozco, S. y Gabbarini, P. A. (2014). Claves pedagógicas que guían la práctica educativa de una maestra. Barcelona: Universitat de Barcelona. Recuperado de http://diposit.ub.edu/dspace/ bitstream/2445/50764/1/Esbrina-Aprender docente p275-281.pdf

Piussi, A. (2013). Volver a empezar. Entre vida, política y educación: prácticas de libertad y conflictos fecundos. Duoda Estudis de la Diferència Sexual, 45, 22-35. Recuperado de http://www.raco.cat/index.php/DUODA/ article/view/271403/359032

Porlán, R., Martín del Pozo, R. y Rivero, A. (1998). Conocimiento profesional y epistemología de los profesores I: estudios empíricos y conclusiones. Enseñanza de las Ciencias, 16(2), 155-171. Recuperado de http://ddd.uab.cat/pub/edlc/021 24521v15n2/02124521v15n2p155.pdf

Secretaría de Educación del Distrito (SED) (2013). Proyecto 901. Prejardín, jardín y transición: preescolar de calidad en el sistema educativo oficial. Bogotá: SED.

Tardif, M. (2009). Los saberes del docente y su desarrollo profesional. Madrid: Narcea.

Torres, A. (1995). Enfoques cualitativos y participativos en la investigación social. Bogotá: Unisur.

Woods, P. (1987). La escuela por dentro: la etnografía en la investigación educativa. Madrid: Paidós-MEC.

Zabalza, M. A. (1988). Condiciones metodológicas en el estudio del pensamiento del profesor. Los autoinformes. En C. Marcelo, Avances en el estudio del pensamiento de los profesores (pp. 9-42). Sevilla: Servicio de Publicaciones de la Universidad de Sevilla. 